L'évaluation en science juridique : retour sur une querelle théorique à propos de la notion de gouvernement des juges

\title{
Gérard Timsit
}

\section{(2) OpenEdition \\ Journals}

Édition électronique

URL : http://journals.openedition.org/ress/196

DOI : $10.4000 /$ ress. 196

ISSN : $1663-4446$

\section{Éditeur}

Librairie Droz

\section{Édition imprimée}

Date de publication : 2 juillet 2007

Pagination : 103-114

ISBN : 2-600-01170-9

ISSN : 0048-8046

\section{Référence électronique}

Gérard Timsit, «L'évaluation en science juridique : retour sur une querelle théorique à propos de la notion de gouvernement des juges ", Revue européenne des sciences sociales [En ligne], XLV-138 | 2007, mis en ligne le 01 juillet 2010, consulté le 21 avril 2019. URL : http://journals.openedition.org/ress/196 ; DOI : 10.4000/ress.196 


\section{L'ÉVALUATION EN SCIENCE JURIDIQUE: RETOUR SUR UNE QUERELLE THÉORIQUE A PROPOS DE LA NOTION DE GOUVERNEMENT DES JUGES}

L'évaluation est placée au cœur de la science juridique de deux manières:par la place que tient l'évaluation dans le domaine de la responsabilité; - et par celle qu'elle a naturellement dans le domaine de la légalité. Dans le premier cas, l'évaluation porte au moins sur la détermination de la part de responsabilité que prend l'auteur d'un fait quelconque aux dommages causés à un individu quelconque: problèmes de la causalité ${ }^{1}$ et de la réparation ${ }^{2}$ du fait dommageable. Dans le second, l'évaluation consiste en la détermination du degré de régularité d'une norme donnée au regard d'une autre norme considérée comme lui étant hiérarchiquement supérieure: problème de la nullité ou de l'annulation de l'acte illégal. Dans les deux cas, l'évaluation consiste en une confrontation, dans le premier, des faits aux normes qui en régissent l'accomplissement; dans le second, des normes inférieures aux normes supérieures qui en régissent l'édiction et l'exécution. On s'en tiendra ici, et pour préserver l'éventuelle originalité d'une investigation dans le domaine de la science juridique, à une analyse de l'évaluation appliquée à la légalité. Dans ce seul domaine en effet, et compte tenu de la nature du système juridique - un système de normes, comme l'a montré Kelsen -, l'évaluation porte exclusivement sur des objets normatifs: des normes inférieures comparées à des normes supérieures. Elle échappera donc, peut-on penser a priori, aux parasitages ou interférences qui résulteraient inévitablement des difficultés de comparaison entre des objets ou faits hétérogènes, normatifs et matériels. Ce n'est pas à dire que les conclusions que l'on tirerait de l'analyse dans l'un et l'autre de ces domaines, légalité et responsabilité, seraient nécessairement différentes. Elles en seraient simplement, faute d'avoir pu faire le départ et le tri entre les mécanismes d'évaluation selon qu'ils fonctionnent exclusivement ou non entre objets normatifs, moins authentiquement et moins purement liés à la spécificité de la science juridique.

Pour mener cette enquête sur l'évaluation dans le domaine de la légalité, on reviendra sur une querelle théorique qui, régulièrement, resurgit chez les juristes et les divise profondément: elle a trait à la notion de gouvernement des juges. C'est une notion à connotation péjorative, en général utilisée de manière polé-

\footnotetext{
1 Problèmes du caractère direct ou indirect des causes du dommage et de l'enchaînement des différentes causes; problèmes de la complexité et de la participation plus ou moins lointaine à l'accomplissement du fait ayant causé le dommage.

2 Problèmes de la prise en compte, dans l'évaluation du dommage, d'éléments autres que matériels et facilement quantifiables : «pretium doloris», atteinte à la réputation; problèmes de reconstitution de carrière pour un fonctionnaire illégalement discriminé ou révoqué, etc.
} 
mique dans le débat juridico-politique pour disqualifier des juges (le plus souvent des juges constitutionnels, des juges suprêmes...) alors accusés d'empiéter abusivement, dans les jugements qu'ils rendent, sur des fonctions qui ne sont pas les leurs - mais celles des gouvernants, exécutif ou législatif, dont ils devraient normalement se contenter de mettre en œuvre les décisions pour les appliquer aux cas singuliers qui leur sont soumis. Les juges sont, assure-t-on, autorisés à contrôler la régularité des lois; ils ne sont pas autorisés à faire plus que cela et à élaborer, sous prétexte d'en interpréter la signification, des normes nouvelles qui vont au-delà de la loi qu'ils sont chargés de faire respecter, et ce faisant, de mettre à exécution. Les juges ne doivent ni ne peuvent donc, conclut-on, se substituer au souverain. Les juges ne doivent pas gouverner.

On voit bien qu'une telle notion a trait à la légalité, -et qu'elle a trait à l'évaluation de la légalité. D'abord apparue aux Etats-Unis (elle trouve son origine chez un juge américain qui lance la formule dans les années 1910 pour désigner la prépondérance des juges sur les deux autres branches du pouvoir, législative et exécutive, dans le fonctionnement empirique du régime politique de son pays), puis importée en France, la formule y est introduite par Édouard Lambert en 1921 et sert de titre à l'ouvrage célèbre qu'il écrit sur les États-Unis: Le gouvernement des juges et la lutte contre la législation sociale: l'expérience américaine du contrôle judiciaire de la constitutionnalité des lois (Paris, Giard, 1921).

À partir de ce moment, la formule est régulièrement reprise dans les débats et les écrits politico-juridiques, et pas seulement en France ou aux Etats-Unis - en général pour, après que l'on ait condamné l'idée même d'un gouvernement des juges, s'inquiéter que, dans les faits, l'on puisse assister à son possible avènement dans un pays déterminé, ou se féliciter au contraire d'y avoir échappé. Il est évident que, dans les deux hypothèses, le recours à la notion de gouvernement des juges implique de la part de ses utilisateurs une double appréciation sur 1) ce que fait un juge, et 2) ce en quoi consiste pour lui le fait de gouverner. La double réponse à cette question implicite est que le juge applique la loi, et que le gouvernement des juges survient quand, au lieu d'appliquer la loi, le juge l'élabore et la crée. Ce qui revient à s'interroger sur les modalités de la relation du jugement à la loi, de la norme juridictionnelle à la norme législative: dans quelle mesure la norme édictée par le juge, la norme juridictionnelle, reste-t-elle conforme à la norme législative qu'il est chargé d'exécuter et qui doit fonder son jugement? De la réponse à cette question dépend la réponse à la question de la reconnaissance de l'existence, ou non, d'un gouvernement des juges. Si le juge reste dans les limites de la loi et qu'il en exécute strictement les dispositions, il ne gouverne pas. Si en revanche, prétextant de son application, il sort des limites de la loi qu'il est chargé d'appliquer, alors il se met à gouverner. Mais, et c'est là tout le problème de la relation du jugement à la loi, la marge d'incertitude qui affecte le contenu de la loi, l'indétermination substantielle de la signification dont sont porteurs les textes normatifs, rend difficile sinon impossible une réponse univoque à la question des conditions dans lesquelles le jugement reste conforme à la loi. L'appréciation de la légalité que suppose le recours à la notion de gouvernement des juges, et les évaluations qu'elle requiert de ce fait, connaissent de sérieux troubles. Mon hypothèse est, à cet égard, que ces troubles tiennent aux conceptions inexactes de la loi et du droit sur lesquelles sont fondées les analyses relatives au gouvernement des juges. Tributaires en effet de l'état actuel de la science et de la théorie juridiques, 
ces analyses ignorent ou passent à côté de ce fait fondamental que la loi n'est qu'un double, un double approximatif, de la réalité qu'elle doit régir. Elles s'en trouvent du coup dans l'impossibilité de procéder correctement aux évaluations qu'appelle normalement l'utilisation de la notion de gouvernement des juges. Il en résulte que le traitement nécessaire des troubles de l'évaluation (I) ne peut passer que par la prise en compte de l'exacte relation entre la législation et ses doubles (II).

\section{LES TROUBLES DE L'ÉVALUATION}

Ils sont d'une extrême diversité et traduisent tous, d'une manière ou d'une autre, la difficulté ou même l'impossibilité, de procéder à une appréciation sûre de l'existence, ou non, dans une situation empirique déterminée, d'un gouvernement des juges. Ces troubles relèvent de deux postures théoriques distinctes. La première est axée sur le contenu de la réponse apportée dans la doctrine juridique à la question du gouvernement des juges. On constatera facilement qu'aucun accord n'existe sur les critères utilisés pour décider de la reconnaissance de l'existence (ou de l'inexistence) d'un tel gouvernement - ce qui peut en effet justifier quelque scepticisme sur l'usage qui est fait aujourd'hui de la notion «évaluatrice» de gouvernement des juges. La seconde posture, quant à elle, tire les conséquences de ce désaccord et du scepticisme qu'il produit, Affichant alors un scepticisme encore plus radical, elle consiste à s'interroger, cette fois, non plus tant sur le contenu de la réponse à apporter à la question du gouvernement des juges, que sur le statut même de la question posée. La question, dit-on alors, n'a pas à être posée, ou en tout cas pas dans ces termes, et pas dans ce but - nouveau trouble de l'évaluation: ce n'est plus la difficulté de l'évaluation qui est relevée, mais la possibilité même de l'évaluation qui est contestée.

\section{La première posture: le contenu de la question, ou les difficultés de l'évaluation}

On a donc affaire avec la notion de gouvernement des juges à une notion qui suppose une évaluation - et donc des critères d'évaluation. Les tenants de cette première posture théorique considèrent en effet que la question posée est susceptible de recevoir une réponse adéquate sur la base de critères objectivement déterminables. Le problème, cependant, surgit avec l'extrême diversité, et la fragilité, des critères utilisés pour apporter une réponse éventuelle à la question. Ils ne coïncident, ces critères, ni quant à l'extension qu'ils confèrent respectivement aux situations en lesquelles on reconnaît un gouvernement des juges; ni quant aux pouvoirs dont l'utilisation éventuelle par des juges permettrait de les faire reconnaître comme des juges qui gouvernent; ni, enfin, quant aux domaines, purement politique ou strictement juridique, qu'il faudrait considérer pour décider si, oui ou non, des juges se sont mêlés de gouverner. 


\section{A. Les difficultés de l'évaluation (I): \\ l'extension du gouvernement des juges}

On a pu distinguer ${ }^{3}$, en fonction des critères adoptés, trois extensions possibles du gouvernement des juges, et par conséquent de la notion appelée à en rendre compte.

Le premier critère confère l'extension la plus large à la notion de gouvernement des juges. Gouverne le juge qui, quel que soit son niveau, constitutionnel ou non, au lieu de juger sur la base stricte de la légalité (constitution ou loi ordinaire), se prononce en opportunité (et non en droit) sur les affaires dont il a à traiter. Il fait la loi (ou la constitution) au lieu de se contenter de la mettre en œuvre.

Le deuxième critère confère à la notion son extension la plus étroite, et pratiquement la réduit à néant. Ne peut, dans cette extension, gouverner que le juge qui s'arroge le pouvoir dévolu normalement au souverain. Or le souverain, dans un pays démocratique en tout cas, peut à tout moment, dans l'hypothèse où un juge sortirait de ses compétences du fait de l'interprétation qu'il conférerait à la loi et qui ne serait pas conforme à la volonté de son auteur, obliger le juge à respecter en définitive sa volonté: «(...) à tout moment [en effet], le souverain, à condition de paraître en majesté, comme constituant, peut dans une sorte de lit de justice, briser leurs arrêts: (...) Par suite, le gouvernement des juges n'existe pas (...) dès lors que le peuple ou ses représentants expriment seuls la volonté souveraine» ${ }^{4}$.

Le troisième critère confère une extension moyenne au phénomène en en limitant le champ aux seuls juges constitutionnels : ne gouvernent, selon cette analyse, que «les juges constitutionnels qui, au nom de la constitution, mais en réalité sans rapport avec les principes qu'elle énonce, condamnent les lois ordinaires votées par la majorité politique du moment et substituent ainsi leur propre volonté à celle du législateur qui a voté et voulu la loi. Sous une apparence usurpée de justice et de règles de droit, ce serait en réalité le subjectivisme le plus désordonné: le droit naturel aurait retrouvé son paradis $»^{5}$.

\section{B. Les difficultés de l'évaluation (II): \\ les pouvoirs d'un gouvernement des juges}

Les pouvoirs utilisés par les juges et le cadre dans lequel ils s'exercent constituent un autre des critères dont se sert la doctrine pour décider si, oui ou non, les juges gouvernent. En général, deux pouvoirs sont considérés de manière privilégiée. Ils ont trait tous deux à ce qui est perçu comme étant de l'essence de l'exercice de leur fonction juridictionnelle et sont tous deux invoqués pour conclure que, décidément, selon que les juges en usent ou non, ils peuvent être, ou pas,

3 On s'inspirera ici, en ne tenant pas compte des multiples variantes introduites par Michel Troper, de la distinction qu'il propose entre trois définitions possibles de la notion in: S. Brondel, N. Foulquier et L. Heuschling (sous la direction de), Gouvernement des juges et démocratie, Publications de la Sorbonne, 2001

4 Georges Vedel, cité in: J. Gicquel, Droit constitutionnel et institutions politiques, Paris, Montchrestien, $15^{\mathrm{e}}$ ed. 1997, p. 306.

5 Ch. Eisenmann, cité par: M. Troper et O. Pfersmann, Existe-t-il un concept de gouvernement des juges? in S. Brondel, N. Foulquier et L. Heuschling (sous la direction de), Gouvernement des juges et démocratie, précité. 
accusés de gouverner - car ils constituent et le signe et le facteur de la discrétionnarité affectant l'exercice d'une fonction. Il s'agit d'une part du pouvoir d'autosaisine - un juge, dit-on, ne peut se saisir lui-même d'une affaire contentieuse. Il ne peut qu'être saisi par un recours émanant d'un justiciable ou une quelconque instance habilitée à cet effet. Si donc un juge se saisit lui-même d'une affaire, en toute autonomie, il s'arroge une prérogative dont ne dispose jamais un juge: il peut dès lors, ce juge, être en effet accusé de gouverner. L'autre pouvoir a trait à la motivation des décisions. Relevant, inconsciemment, d'une conception schmittienne du pouvoir, - le souverain, c'est celui qui décide de l'exception -, elle consiste à reconnaître le pouvoir de gouverner à celui qui n'a pas à motiver sa décision. Or, dit-on, non seulement les juges ont à argumenter pour justifier leur jugement, mais il existe même une spécificité argumentative ${ }^{6}$ de la fonction juridictionnelle - la forme syllogistique, par exemple - qui la distingue fondamentalement des motivations dont, de plus en plus, et malgré le caractère souverain de la décision publique, celle-ci tend à s'entourer pour se rendre plus acceptable, partant, plus légitime, et par conséquent, en fin de compte, plus efficace.

\section{Les difficultés de l'évaluation (III): le domaine du gouvernement des juges}

Les critères invoqués pour décider de l'existence, ou non, d'un gouvernement des juges peuvent enfin relever d'une troisième catégorie. Très grossièrement constituée, cette troisième catégorie est fondée sur la distinction du politique et du juridique et ferait alors du gouvernement des juges le fait pour un juge d'empiéter sur les compétences du pouvoir politique et d'entrer ainsi dans un domaine qui n'est pas le sien: il existerait donc des compétences «politiques » par nature - la détermination des principes fondamentaux qui régissent une société, le devoir de trancher entre les contradictions qu'ils recèlent et de définir avec exactitude leur portée, par exemple. - des compétences qui, si le juge les exerçait ou avait à le faire, feraient de lui non plus un juge mais un gouvernant.

On voit la fragilité de tous ces critères et des évaluations qu'ils prétendent autoriser et fonder. Leur extrême multiplicité, d'abord, témoigne du désaccord fondamental qui règne aujourd'hui dans la science juridique sur les moyens d'évaluer des normes au regard les unes des autres - signe, au moins, du caractère incertain des bases sur lesquelles est établie la science juridique dans son état actuel. On pourrait, cependant, vouloir penser qu'il ne s'agit que d'approches différentes du phénomène du gouvernement des juges. Encore faudrait-il que le recours aux critères relevant des différentes catégories n'aboutisse pas à des résultats contradictoires, et que ne fussent qualifiés de gouvernement des juges, au titre de l'un des critères, des phénomènes qui ne pourraient se voir reconnaître cette qualification au titre de l'un des autres: or, non seulement, les différentes notions de gouvernement des juges ne coïncident pas, mais ne se recoupent partiellement

6 Voir D. de Bechillon (in S. Brondel, N. Foulquier et L. Heuschling (sous la direction de), précité pp. 341-355), par exemple, qui s'inquiète de ce que le recours à la notion de gouvernement des juges ne pourrait, dit-il, permettre de «penser la spécificité argumentative » de la fonction juridictionnelle. 
même pas toujours ${ }^{7}$. De surcroît, les critères devraient être moins grossièrement taillés qu'ils ne sont - les catégories qu'ils constituent étant de ce fait trop floues pour être facilement utilisables - le critère du politique, entre autres, paraissant à cet égard particulièrement sujet à caution. On peut donc comprendre que certains auteurs en soient venus à adopter une autre posture théorique et se soient interrogés, plutôt que sur le contenu de la réponse apportée à la question du gouvernement des juges, sur le statut même à conférer à cette question...

\section{La deuxième posture: le statut de la question, ou l'impossibilité de l'évaluation}

Scepticisme radical: puisque les critères sont si nombreux et si fragiles, c'est qu'il n'y a pas de réponse à la question du gouvernement des juges - que l'évaluation qu'elle suppose est impossible et que, dans ces conditions, la question n'a pas à être posée ou, en tout cas, qu'elle est mal posée. Dans la première hypothèse, on affirme que l'on a affaire à un non-sujet; dans la seconde, que la question est insoluble et qu'elle doit être reformulée. Dans les deux cas, pourtant, l'on verra qu'alors une telle posture nie toute possibilité d'évaluation, elle la présuppose dans le premier cas, et la rend inéluctable dans le second...

A. Un non-sujet ${ }^{8}$ : c'est la position adoptée par ceux - ils sont en général farouchement positivistes - qui, n'ayant pas trouvé de réponse adéquate dans les critères proposés par les différentes théories énoncées et soucieux d'afficher au plus près leur proximité à l'égard des textes qui fondent les jugements rendus - considèrent qu'un juge ne gouverne jamais - et qu'il ne peut jamais gouverner. L'expression "gouvernement des juges », disent-ils, a été inventée ailleurs (c'est vrai: elle l'a été aux Etats-Unis) et abusivement transportée dans un contexte qui n'est pas le sien, ce qui, du coup, lui ôte toute signification. Quand ce sont des juges qui prennent cette posture, ils ont pour souci essentiel d'affirmer leur respect de la répartition des compétences et de la séparation des pouvoirs et de témoigner de leur intention (et de sa réalité prétendue...) de s'en tenir à la mise en œuvre stricte des lois dont ils sont chargés de sanctionner l'inexécution. Ils savent, et pour cause, ces juges, l'indétermination essentielle de ces textes et la liberté qu'elle leur donne dans la détermination de leur signification. Ils affirment donc, à titre de précaution, - pour, a priori, repousser et contester la légitimité de toute interrogation qui viendrait à mettre en doute leur soumission proclamée aux textes - qu'en tout état de cause ils font preuve dans l'exercice de leurs fonctions de ce que les

7 Ainsi, un juge qui, au titre du critère 1.C - un juge constitutionnel qui annulerait une loi ordinaire - serait accusé de tomber dans le gouvernement des juges, serait exonéré d'une telle condamnation au titre, par exemple des critères 2.A et 2.B (il ne s'est pas saisi lui-même de l'affaire dans laquelle il a eu à se prononcer ni n'a manqué d'argumenter de manière apparemment aussi rigoureusement syllogistique que le juge le plus respectueux des textes constitutionnels qu'il est chargé de mettre en œuvre...)

8 Sur ce point, voir Louis Favoreu, in S. Brondel, N. Foulquier et L. Heuschling (sous la direction de), précité p. 288. 
Américains appellent judicial self restraint - de la «réserve judiciaire ${ }^{9}$ : une «autodiscipline évitant aux juges de faire prévaloir des opinions personnelles qui sont contraires aux précédents existants ou aux lois $»^{10}$.

Quand ce sont des universitaires qui se rallient à cette position, ils remarquent aussi, et se contentent parfois de cette remarque, que la notion de gouvernement des juges n'a pu finalement revêtir cette signification d'un excès de pouvoir que dans des pays qui, comme la France jusqu'en 1958, n'avaient pas de juridiction constitutionnelle et n'avaient par conséquent pas de juges qui fussent mêlés de si près à l'élaboration des lois - fonction politique par excellence ${ }^{11}$. Dans la réalité, vous assurent-ils, le gouvernement des juges n'existe donc pas, et ne peut exister - et la notion de gouvernement des juges n'est que le produit d'une illusion d'optique. L'évaluation est donc impossible - parce qu'elle est inutile.

B. Ce n'est pas ce que disent d'autres théoriciens qui, admettant pourtant, eux aussi, l'impossibilité de toute évaluation, considèrent que, certes, la question se pose, mais qu'elle est insoluble et que, dans ces conditions, elle doit être posée autrement. L'un des tenants de cette analyse est très clair sur ce point. Relevant que toutes les définitions du gouvernement des juges envisagées jusqu'à présent s'inscrivent dans le cadre de la question: les juges gouvernent-ils, ils les écartent toutes parce qu'elles dépendent toutes, dit-il, «seulement de la décision prise au sujet de cette définition ». Toutes les définitions étant en effet nécessairement stipulatives, elles posent a priori les caractères de l'objet qu'elles prétendent définir - « un concept stipulatif ne peut permettre d'établir la vérité d'une thèse, mais seulement déterminer le champ d'une recherche $»^{12}$. Il faut donc, poursuit-il, renoncer à la question insoluble: les juges gouvernent-ils, et lui en substituer une autre: comment les juges gouvernent-ils? C'est une question qui, elle, est susceptible de recevoir des réponses, et des réponses objectives - deux types de réponses, l'une et l'autre possibles en vertu d'une simple description (objective), et non plus d'une évaluation (stipulative), de la réalité: d'une part, les juges gouvernent autrement que les législateurs; d'autre part, les juges ne gouvernent pas tous de la même manière ${ }^{13}$ - ce qui, fondé sur l'affirmation implicite que tous les juges gouvernent, rend nécessaire de remplacer, en fin de compte, la question insoluble du gouvernement des juges par celle du gouvernement par les juges.

Double dérobade: l'une et l'autre des deux analyses - du non-sujet et de la question insoluble- en relèvent pour des raisons symétriques et inverses. La première, parce qu'affirmant qu'aucun juge ne gouverne, elle est fondée sur une conception a priori du jugement qui englobe tous les types de décisions rendues par les juges, même celles qui se situeraient en dehors des limites de leur pouvoir.

${ }^{9}$ Sur ce point, voir Robert Badinter, in S. Brondel, N. Foulquier et L. Heuschling (sous la direction de), précité p. 292.

${ }^{10}$ Définition citée par L. Pech Le remède au gouvernement des juges: le judicial self-restraint? in S. Brondel, N. Foulquier et L. Heuschling (sous la direction de), précité, p. 85, note 99.

\footnotetext{
${ }^{11}$ L. Favoreu, précité.

${ }^{12}$ M. Troper, précité, pp. 30-31.

${ }^{13}$ M. Troper, précité, p. 33
} 
La seconde, parce qu'affirmant que tous les juges gouvernent, elle se donne une conception a priori du gouvernement ${ }^{14}$ qui englobe tous les types de décisions rendues par des juges, même celles qui resteraient à l'intérieur des limites de leur pouvoir... Double dérobade en ce que l'une et l'autre des deux postures impliquent une évaluation qui, dans le premier cas, est présupposée - et qui, dans le second, sera de toute façon, rendue nécessaire au moment où, ayant tiré les conséquences de l'analyse des différentes formes de gouvernement, on devra bien finir par procéder à une comparaison (c'est-à-dire à une évaluation) des modes de gouvernement par les juges et par le législateur...

\section{LA LÉGISLATION ET SES DOUBLES}

On aborde ici une deuxième phase, non plus celle du simple constat de l'existence de troubles dans l'évaluation, mais de la recherche des raisons pour lesquelles se produisent de tels troubles.

1. Mon hypothèse, ai-je dit, est que ces troubles proviennent, en fait, de ce que la doctrine n'a pas su prendre en compte dans ses analyses les dimensions exactes du phénomène normatif. Il en est résulté ces difficultés: la fragilité, ou l'impossibilité - des évaluations difficiles en raison de leur fragilité, ou impossibles du fait de leur brutalité. Ce que je voudrais, c'est essayer donc d'abord de remonter de ces troubles empiriques aux lacunes théoriques qu'ils aident à repérer. Ce qui fait la fragilité des évaluations proposées par la doctrine juridique, on l'a relevé précédemment, ce sont en effet leurs références contradictoires (et leur hésitation constante entre elles, d'un mode d'évaluation à l'autre) à des éléments antagonistes, parfois grossièrement définis : légalité/opportunité; droit/politique; discrétion/subordination... Et ce qui fait leur impossibilité? - au-delà des apparences, les mêmes références contradictoires, la même hésitation, la même rusticité dans des évaluations présupposées a priori ou rendues nécessaires a posteriori. Les troubles de l'évaluation convergent donc tous vers la dénonciation d'une même défaillance fondamentale de la doctrine juridique: la non prise en compte, ou la prise en compte inexacte, des éléments constitutifs de la norme.

On peut certes s'expliquer de telles défaillances. Le positivisme, et avec lui la doctrine moderne du droit, est né de la préoccupation de fonder objectivement la science juridique sur des éléments scientifiquement appréhendables et empiriquement déterminables. D'où la référence à un droit posé - jus positum - par le législateur, le texte de la loi auquel devrait se tenir strictement le juge. On conçoit alors que, sur de telles prémisses, aient pu proliférer des analyses qui, soit à l'un des extrêmes de la doctrine, aient tenté de procéder à une évaluation normative sur la base des seuls textes qui devaient, en bonne doctrine positiviste, fonder l'action des juges, soit, à l'autre extrême, aient au contraire contesté toute tentative d'évaluation qui fût fondée sur ce texte seul. Ainsi s'expliquent, et peuvent être catégorisées, les différentes postures théoriques précédemment recensées. Relèvent de la première catégorie toutes celles qui prétendent distinguer la légalité de l'opportu-

14 Ibid. 
nité et faire le tri entre la politique et le droit - un droit réduit au besoin à des techniques formelles et procédurales comme celles qui ont trait au pouvoir de saisine des juges. Relève également de cette catégorie la posture du non-sujet qui consiste à considérer que, puisque le juge décide en vertu de textes normatifs, la signification qu'il confère à ces textes n'est jamais que celle qu'aurait voulue leur auteur, le législateur souverain, dont le juge en définitive reste alors, selon la formule de Montesquieu, la bouche qui prononce les paroles de la loi.

Relèvent en revanche de la seconde catégorie les postures choisissant, au contraire, de nier tout autre critère que purement politique et de ne reconnaître de gouvernement des juges que dans le comportement de ceux seuls qui, quel que soit le fondement juridique de leur jugement, substituent leur propre volonté à celle du législateur - contestation de la possibilité même d'une justice constitutionnelle. Relève aussi de cette posture la position consistant à déclarer insoluble la question du gouvernement des juges et à vouloir lui substituer celle du gouvernement par les juges: il s'agit là de postuler en effet que, puisque tout juge, qu'il agisse ou non sur la base de textes juridiques, a une action politique, il n'y a guère d'intérêt à chercher sur quel fondement il assoit son action. Ce qui importe, dit-on, ce sont les modalités de son action, et ce qui la distingue de celles des autres instances ayant une action politique.

En définitive, il apparaît que ce n'est pas tant la notion de gouvernement des juges qui doit être contestée que les conditions dans lesquelles elle est utilisée. La notion, elle, désigne une réalité - et de toutes les postures théoriques précédemment recensées, peu d'entre elles en définitive nient qu'une telle réalité puisse effectivement se produire et se rencontrer. En revanche, on peut légitimement s'interroger sur la manière de reconnaître quand et où prend corps une telle réalité. Et sur ce point, force est de constater que les doctrines dominantes dans la science juridique, -le positivisme à l'origine, et la réaction «réaliste» qu'il a provoquéeintroduisent des biais - la fragilité des évaluations - ou font surgir des obstacles insurmontables - leur impossibilité ${ }^{15}$. On ne pourra donc traiter du gouvernement des juges que si l'on dispose d'instruments conceptuels qui ne tombent dans l'un ni l'autre des excès de la doctrine dominante: la réduction abusive du droit au texte de la loi et l'ignorance de la place des valeurs politiques dans sa définition, comme le fait le positivisme, ou à l'opposé l'identification abusive du droit aux valeurs politiques dont on prétend qu'elles le fondent, ce qui est le cas du réalisme. Deux perversions, en fait, de l'analyse qui n'arrive pas à prendre la loi pour ce qu'elle est vraiment: un instrument de traitement de la réalité (elle a une fonction de représentation de la réalité), ni à reconnaître dans le raisonnement juridique et les concepts qu'il utilise la fonction qui est bien la leur: de suppléance de la loi. C'est de cette double déformation qu'introduit la distance de la loi à la réalité, d'une part, et du raisonnement juridique à la loi, d'autre part, que celui-ci tire sa spécificité. Le raisonnement a pour fonction de suppléer à l'indétermination de la loi - qui a pour fonction de «représenter» la réalité. Représentation qui ne peut être la reproduction - elle n'en est que la schématisation nécessaire - d'une réalité

${ }^{15}$ Sur les doctrines dominantes dans la science juridique, cf. G. Timsit, Contre la nouvelle vulgate, in Le nouveau constitutionnalisme, Mélanges en l' honneur de Gérard Conac, Economica 2001; et La loi et ses doubles, thématiques du raisonnement juridique, Droits n 36, 2002, pp. 135-159. 
empirique dont la loi doit assurer le traitement. Paul Ricœur parle, pour désigner une telle fonction de représentation approximative de la réalité, de représentance. Dans une telle fonction de représentance, la loi ne fait jamais qu'abstraire de la réalité ceux des éléments auxquels elle réduit la réalité - sur la base desquels, ensuite, elle procède à sa reconstitution. Ce sont ces éléments qui forment ce que l'on appelle les «conditions pertinentes» au regard desquelles le juge chargé d'appliquer la loi vérifie que, remplies en l'espèce, elles justifient l'application de la loi au cas concret auquel il est confronté. On est là au cœur de l'opération juridique -une opération de qualification des faits. Une telle opération constitue une sorte de test de la manière dont fonctionnent le raisonnement juridique et ses concepts. Et ce que l'on a pu voir, ce sont les théories (et les concepts dont elles font usage - le concept de gouvernement des juges par exemple) se distribuer de part et d'autre de l'axe de la représentance, les unes et les autres de ces théories s'abîmant, selon le cas, du côté d'une sur - ou d'une sous- représentation de la réalité. En deçà ou au delà, les doctrines juridiques font de la loi, soit - c'est le cas des doctrines positivistes - un instrument parfaitement abstrait, coupé de la réalité (la loi y est réduite aux textes normatifs); soit - c'est le cas des doctrines réalistesun outil aussi exactement calqué que possible, croient-elles, sur une réalité qu'il s'agit selon elles de figurer au plus près: ce qui, alors, après avoir donné lieu à l'extension du raisonnement juridique aux valeurs, politiques en particulier, qui commandent à l'interprétation de la loi - débouche sur leur sur-évaluation dans la détermination de sa signification.

Ainsi s'expliquent, à partir d'une telle analyse, les deux types symétriques et inverses de défauts ou défaillances du raisonnement juridique. Deux types de troubles, en effet. Des troubles consistant, soit à ignorer que la loi comporte une part de représentation de la réalité, soit au contraire - tombant d'un excès dans l'autre, en en accentuant jusqu'à la caricature ce que l'on croit devoir être sa fonction - à vouloir faire de la loi un calque de la réalité. Troubles, dans ce dernier cas, de la sur-assimilation, de la sur-représentation, de la sur-figuration, de la réalité; troubles, dans le précédent, de la sur-abstraction ${ }^{16}$. Le raisonnement à l'œuvre, dans cette hypothèse, est typiquement, alors, syllogistique. La logique est une logique de déduction, et les opérations auxquelles se livre le juge y sont analysées comme des opérations de reproduction à l'identique, de [re]transcription, dans la norme inférieure de l'écriture de la norme hiérarchiquement supérieure, sans que l'auteur de la norme subordonnée soit considéré comme se donnant la moindre liberté à l'égard du texte de la loi. Quant à la séquence du raisonnement, c'est une séquence limitée à la production d'une norme à partir de la seule reproduction du contenu de la norme supérieure. Le gouvernement des juges, dans une telle analyse, en devient parfaitement inconcevable. Cela ne peut exister - un non-sujet, vous disait-on. Mais on voit ce qu'il y a d'abstraction, et d'éloignement de la réalité, dans une telle analyse - et l'on comprend comment et pourquoi l'école réaliste voudra, tout à l'opposé, et conformément à la vocation que lui assigne et désigne sa dénomination, renouer avec les réalités. Ce faisant, elle tombera dans

16 J'emprunte à R. Jakobson, Essais de linguistique générale, Ed. de minuit, coll. Points, 1963, p. $43 \mathrm{~s}$. cette distinction entre troubles de la contiguïté de la similarité qu'il applique à l'analyse des troubles du langage, spécialement à l'analyse de l'aphasie. 
un autre piège. Dépassant son objectif, elle s'abîme, du coup, dans les défauts de la sur-figuration de la réalité dont elle prétend traiter à travers la loi. Intégration dans le raisonnement, au prétexte - justifié - de figuration de la réalité, des valeurs politiques commandant à l'interprétation du texte de loi, mais dans des conditions telles qu'elles finissent par l'éclipser. Au point que, désormais, dans le raisonnement, prévaut une logique de discrétion -de discrétionnarité - à l'égard du texte de loi- le juge s'estimant libre (ou la doctrine le considérant comme tel...) de donner à la loi telle interprétation qui lui paraît la plus souhaitable en fonction des valeurs auxquelles il adhère ${ }^{17}$. Non seulement le gouvernement des juges est alors concevable, mais c'est la transgression qui devient la règle.

2. La vérité est que, pour remédier à ces deux types de troubles -et aux excès auxquels peuvent donner lieu les opérations qui leur sont liées de transcription ou de transgression, de subordination ou de contravention à la norme hiérarchiquement supérieure-, se place un autre mode d'opération et de raisonnement, à michemin des deux excès de la sur-abstraction et de la sur-figuration de la réalité: opération délicate, essentielle, de contextualisation de la norme. J'ai parlé de transdiction - un néologisme créé sur le modèle de: juridiction, juris-dictio - pour désigner cette dite du droit - cet énoncé d'un droit singulier à partir d'une règle formulée en termes généraux - à ne confondre avec aucune des deux opérations précédentes. Les variables d'un tel énoncé? La singularisation des faits: la prise en considération par le juge, comme dans l'opération de transcription, des faits singuliers au regard desquels doit être appliquée la norme énoncée en termes généraux et abstraits; et, comme dans l'opération de transgression, mais à la différence de la transcription, la dialogisation de la référence: la prise en compte par le juge de valeurs autres, - relevant d'une autre logique- que celles auxquelles se réfère, implicitement ou explicitement, le Législateur auteur de la loi. L'une et l'autre des variables, combinées, sont destinées à assurer l'adéquation de la règle générale au contexte singulier dans lequel elle est appliquée, sa déclinaison -au sens grammatical du terme- en fonction des cas à $\operatorname{traiter}^{18}$. La singularisation des faits et la dialogisation de la référence, associées, si elles permettent de dépasser le stade de la simple (re)transcription monologique de la règle qu'il s'agit pour le juge d'appliquer, ne vont cependant jamais jusqu'à la transgression de la règle au nom des réalités. Difficile équilibre de la transdiction, qui n'est guère pourtant que l'opération la plus caractérisée d'une conception émergente et plus appropriée aux réalités empiriques de la normativité. Une conception théorique qui, reconnaissant la place des valeurs dans la détermination de la signification de la loi, permette de ne pas penser seulement la transcription du texte de la loi dans les normes inférieures, comme le font les positivistes - qui, de ce fait, en restent incapables de penser le gouvernement des juges et n'ont jamais réussi à découvrir un juge qui

${ }^{17}$ On connaît la formule caricaturale, et révélatrice, qui résume cette position: «le droit, c'est ce que le juge a mangé à son petit-déjeuner...»

18 Sur ces variables, voir les deux études de cas consacrées l'une à la jurisprudence sur l'erreur manifeste d'appréciation et la théorie du bilan (G. Timsit, Guy Braibant, un juge qui gouverne? in L'Etat de droit, Mélanges en l' honneur de Guy Braibant, Dalloz 1993) et l'autre à la jurisprudence sur l'extradition (G. Timsit, La transdiction, étude de cas, in Les objets du droit, Enquêtes, $\mathrm{n}^{\circ} 7$ ). 
se soit jamais laissé aller à gouverner. Une conception théorique qui, ne reconnaissant pas de place exclusive aux valeurs dans la détermination de la signification de la loi, permette de ne pas penser seulement la transgression du texte de la loi (comme le font les réalistes, qui du coup, en renoncent à penser toute autre situation que celle du gouvernement des juges et ne voient autour d'eux que des juges qui gouvernent...). Une conception qui par cette place, non exclusive, qu'elle reconnaît aux valeurs dans le droit, peut penser à la fois et les différentes figures du jugement, et les évaluations qu'elles supposent - de la transcription à la transgression, en passant par les différentes formes non transgressives de l'interprétation que j'ai appelées transdiction. Une conception théorique qui permette de penser en définitive non seulement les différentes figures du jugement, mais aussi celles des positions et postures théoriques qui prétendent pouvoir en rendre compte. Ou qui y échouent - ou qui y renoncent.

Université de Paris-I Panthéon Sorbonne 